\title{
Universal Fast-Flux Control of a Coherent, Low-Frequency Qubit
}

\author{
Helin Zhang $\odot,{ }^{1,2}$ Srivatsan Chakram, ${ }^{1,2}$ Tanay Roy $\odot,{ }^{1,2}$ Nathan Earnest, ${ }^{1,2, \dagger}$ Yao Lu $\odot,{ }^{1,2, \sharp}$ Ziwen Huang $\odot,{ }^{3}$ \\ D. K. Weiss, ${ }^{3}$ Jens Koch, ${ }^{3}$ and David I. Schuster ${ }^{1,2,4, *}$ \\ ${ }^{1}$ James Franck Institute, University of Chicago, Chicago, Illinois 60637, USA \\ ${ }^{2}$ Department of Physics, University of Chicago, Chicago, Illinois 60637, USA \\ ${ }^{3}$ Department of Physics and Astronomy, Northwestern University, Evanston, Illinois 60208, USA \\ ${ }^{4}$ Pritzker School of Molecular Engineering, University of Chicago, Chicago, Illinois 60637, USA
}

(Received 4 March 2020; revised 21 October 2020; accepted 2 December 2020; published 15 January 2021)

\begin{abstract}
The heavy-fluxonium circuit is a promising building block for superconducting quantum processors due to its long relaxation and dephasing time at the flux-frustration point. However, the suppressed charge matrix elements and low transition frequency make it challenging to perform fast single-qubit gates using standard protocols. We report on new protocols for reset, fast coherent control, and readout that allow highquality operation of the qubit with a $14 \mathrm{MHz}$ transition frequency, an order of magnitude lower in energy than the ambient thermal energy scale. We utilize higher levels of the fluxonium to read out the qubit state and to initialize the qubit with $97 \%$ fidelity corresponding to cooling it to $190 \mu \mathrm{K}$. Instead of using standard microwave pulses, we control the qubit only with fast-flux pulses, generating control fields much larger than the qubit frequency. We develop a universal set of gates based on nonadiabatic Landau-Zener transitions that act in 20-60 ns, less than the single-qubit Larmor period. We measure qubit coherence of $T_{1}, T_{2 e} \sim 300 \mu$ s for a fluxonium in a $2 \mathrm{D}$ architecture and realize single-qubit gates with an average gate fidelity of $99.8 \%$ as characterized by randomized benchmarking.
\end{abstract}

DOI: 10.1103/PhysRevX.11.011010

\section{INTRODUCTION}

Superconducting circuits are among the fastest-developing candidates for quantum computers due to steady improvements in coherence times, gate fidelities, and processor size over the past two decades [1,2]. These developments have ushered the noisy intermediate-scale quantum era [3] and demonstrations of quantum advantage over classical computing [4]. Despite these significant improvements, decoherence still remains the central challenge for superconducting circuits. While the effects of environmental noise can be reduced by improving material properties [5-7], here, we develop an alternative approach in which we drastically reduce the noise spectral density by lowering the qubit

\footnotetext{
* Corresponding author.

David.Schuster@uchicago.edu

${ }^{\dagger}$ Present address: IBM T. J. Watson Research Center, Yorktown Heights, New York 10598, USA.

*Present address: Department of Applied Physics, Yale University, New Haven, Connecticut 06511, USA.

Published by the American Physical Society under the terms of the Creative Commons Attribution 4.0 International license. Further distribution of this work must maintain attribution to the author(s) and the published article's title, journal citation, and DOI.
}

Subject Areas: Atomic and Molecular Physics

Condensed Matter Physics

Quantum Information frequency from gigahertz to megahertz. Despite the slowdown of all the relevant timescales, we still perform singlequbit gates at speeds that are comparable to the state of the art in conventional qubits. We develop ultrafast gates based on nonadiabatic Landau-Zener transitions [8-12] which act in less than a single Larmor period.

We achieve this result by using a heavy-fluxonium circuit $[13,14]$, whose rich level structure features a low qubit frequency (approximately $14 \mathrm{MHz}$ ) and excited levels that are several gigahertz away. The large frequency separation enables us to apply flux drives with amplitudes much larger than the qubit frequency, without any leakage outside of the computational subspace. The gap to the excited levels also exceeds the frequency scale set by the ambient temperature, allowing them to be used for initialization and readout of the qubit state. We develop a completely new set of protocols for operating the heavy fluxonium, demonstrating state-of-the-art performance that makes it the first serious competitor to the transmon qubit [15], with the potential for further improvements.

The transmon, which underlies most modern superconducting quantum processors, is a weakly anharmonic oscillator with large dipole matrix elements. This circuit trades off increased sensitivity to decay and a reduced anharmonicity $\alpha$ for decreased sensitivity to charge-noiseinduced dephasing. Despite the maximal susceptibility to 
relaxation, state-of-the-art transmons have depolarization $\left(T_{1}\right)$ times around $100 \mu \mathrm{s}[7,16,17]$, corresponding to quality factors $Q$ of a few million. The gate speeds are, however, limited by the small anharmonicity, typically approximately $5 \%$ of the qubit frequency $\omega_{q}$, resulting in a theoretical upper bound for the gate infidelity of approximately $\omega_{q} /(Q \alpha) \sim 10^{-5}$ and state-of-the-art values of $\lesssim 1-2 \times 10^{-4}$ [4] achieved with the derivative removal by adiabatic gate or other shaped pulses. This value suggests that gate infidelities can approach $1 / Q$ by increasing the anharmonicity in comparison to qubit frequency and performing gate operations within a few Larmor periods.

The flux qubit [18-21], another member of the superconducting circuit family, already has the desired level structure with a relative anharmonicity $\alpha / \omega_{q} \gg 1$. The extreme sensitivity to flux noise of these qubits is mitigated by shunting the Josephson junction with a large superinductor, resulting in the development of the fluxonium [22-25]. Further improvements in energy relaxation times were obtained by the realization of a heavy fluxonium $[13,14]$, which additionally reduces the decay matrix elements using a large shunting capacitor. These variants of the fluxonium are reported to have longer coherence times than transmons in 3D architectures [26]. Even though the heavy fluxonium has the desired level structure and large coherence times, fast manipulation of the metastable qubit states remains a challenge due to the suppressed charge matrix elements. While Raman transitions can be used for coherent operations [13,27], these protocols are still relatively slow and require high drive powers while exposing the qubit to the higher loss rates of excited fluxonium levels involved during the gate. The requirement of fast coherent control, thus, encourages one to explore new schemes for implementing gates.

In this work, we realize a heavy-fluxonium circuit in a 2D architecture with coherence times $T_{1}, T_{2 e} \sim 300 \mu \mathrm{s}$ exceeding those of standard transmons. The long coherence is achieved by accessing a new regime of low qubit frequency, which has the effect of slowing down all decoherence processes. Our circuit has a gap of only $14 \mathrm{MHz}$, an order of magnitude lower than the temperature of the surrounding bath. Therefore, we develop and realize a reset protocol that utilizes the readout resonator and higher circuit levels to initialize the qubit with $97 \%$ fidelity, effectively cooling the qubit down to $190 \mu \mathrm{K}$. Despite a frequency that is 300 times smaller, we develop an ultrafast-flux gate protocol that performs single-qubit gates at similar speeds to that of typical transmons.

\section{THE HEAVY-FLUXONIUM CIRCUIT}

The circuit consists of a small-area Josephson junction (JJ), with inductance $L_{J}$ shunted by a large inductance $\left(L_{J A}\right)$, and a large capacitor $\left(C_{q}\right)$, as shown in Fig. 1(a). The shunting inductance is realized by an array of 300 large-area JJs, each having a Josephson energy $E_{J A}$ and charging energy $E_{C A}$. We make $E_{J A} / E_{C A} \gg 1$ to ensure that the charge dispersion for each array junctions is small, and the array can be regarded as a linear inductor. The corresponding effective circuit is shown in Fig. 1(b), resulting in a Hamiltonian of the form

$H_{f}=-4 E_{C} \frac{d^{2}}{d \varphi^{2}}-E_{J} \cos \left(\varphi-2 \pi \frac{\Phi_{\mathrm{ext}}}{\Phi_{0}}\right)+\frac{1}{2} E_{L} \varphi^{2}$,

where $E_{C}=e^{2} /\left(2 C_{q}\right)$ is the charging energy, $E_{J}=$ $\Phi_{0}^{2} /\left(4 \pi^{2} L_{J}\right)$ the Josephson energy of the small junction, and $E_{L}=\Phi_{0}^{2} /\left(4 \pi^{2} L_{J A}\right)$ the inductive energy of the JJ array. $\Phi_{\text {ext }}$ denotes the flux threading the loop formed by the small junction and the superinductance, and $\Phi_{0}$ is the quantum of flux. The corresponding values for the reported device are $E_{C} / h=0.479 \mathrm{GHz}, E_{L} / h=0.132 \mathrm{GHz}$, and $E_{J} / h=3.395 \mathrm{GHz}$, where $h$ is Planck's constant. The level structure of the fluxonium at the flux-frustration point $\left(\Phi_{\text {ext }}=\Phi_{0} / 2\right)$ is shown in Fig. 1(c). There are two types of transitions of interest: the intrawell plasmons $(|g\rangle \leftrightarrow|h\rangle$ and $|e\rangle \leftrightarrow|f\rangle)$ and interwell fluxons $(|g\rangle \leftrightarrow|e\rangle$ and $|f\rangle \leftrightarrow|h\rangle$ ). Since the wave functions of $|g\rangle$ and $|f\rangle$ are both even in phase space, and $|e\rangle$ and $|h\rangle$ are both odd, the single-photon transitions $|g\rangle \leftrightarrow|f\rangle$ and $|e\rangle \leftrightarrow|h\rangle$ are forbidden at the flux-frustration point due to the parity

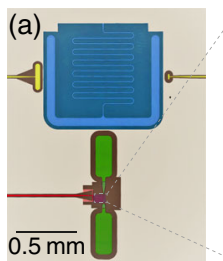

(b)

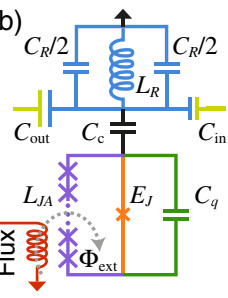

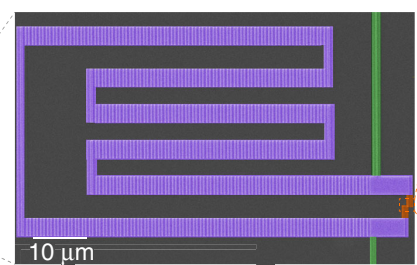

(c)

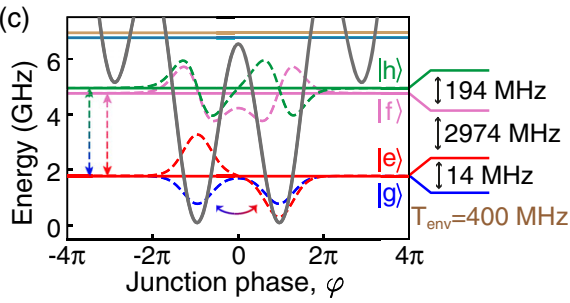

FIG. 1. Device, circuit, and energy-level diagram. (a) Left: False-colored optical microscope image of the fluxonium coupled to a readout resonator (blue) along with flux (red) and inputoutput (yellow) lines. Middle: Scanning electron micrograph of the large junction array (purple) and the small Josephson junction (orange). Right: Enlarged view of the small junction. (b) Circuit diagram for the heavy-fluxonium qubit. (c) Energy-level diagram of the heavy fluxonium at the flux-frustration point $\left(\Phi_{\text {ext }}=\right.$ $\left.\Phi_{0} / 2\right)$. The gray line represents the potential well. The first six energy eigenstates are depicted by the colored lines; dashed lines show the wave functions for the first four levels with corresponding color. The fluxonlike transition $|g\rangle \leftrightarrow|e\rangle$ is marked with a solid arrow, while plasmonlike transitions $|g\rangle \leftrightarrow|h\rangle$ and $|e\rangle \leftrightarrow|f\rangle$ are marked with dashed arrows. 
selection rule. The qubit is comprised of the lowest two energy levels $|g\rangle$ and $|e\rangle$, with the qubit transition being fluxonlike, with a frequency of $14 \mathrm{MHz}$.

\section{QUBIT INITIALIZATION AND READOUT}

Because of its low transition frequency, the qubit starts in a nearly evenly mixed state in thermal equilibrium. We first initialize the qubit in a pure state $(|g\rangle$ or $|e\rangle)$ using the reset protocol shown in Fig. 2(a), similar to Refs. [28,29]. In this protocol, we simultaneously drive both the $|g 0\rangle \rightarrow|h 0\rangle$ and $|h 0\rangle \rightarrow|e 1\rangle$ transitions for $15 \mu \mathrm{s}$. The high resonator frequency $(5.7 \mathrm{GHz})$, in comparison to the physical temperature, and the low resonator quality factor $Q=$ 600 result in the rapid loss of a photon from $|e 1\rangle$, effectively removing the entropy from the qubit. In conjunction with the large matrix element between $|h 0\rangle$ and $|e 1\rangle$, this loss steers the system into a steady state with approximately $97 \%$ of the population settling in $|e 0\rangle$ in $15 \mu$ s (see the Appendix E). We subsequently perform an additional $\pi$ pulse on the $|g\rangle-|e\rangle$ transition to initialize the system in the ground state $(|g 0\rangle)$. The reset is characterized by performing a Rabi rotation between the $|e\rangle \leftrightarrow|f\rangle$ levels, as shown in Fig. 2(b). The Rabi contrast is doubled following reset, consistent with approximately $50 \%$ of the population being in $|e\rangle$ in thermal equilibrium. If we prepare the system in $|g\rangle$, the $|e\rangle \leftrightarrow|f\rangle$ Rabi contrast indicates a $3 \pm 2 \%$ error in state preparation, depending on the $|f\rangle$ state thermal population. Since the $|f\rangle$ frequency is similar to the typical transmon frequencies, its thermal population is in line with that of most transmons. The effective qubit temperature following reset is approximately
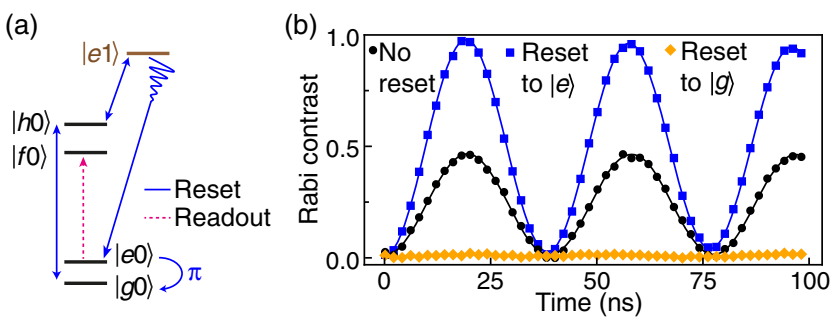

FIG. 2. Reset and readout schemes and measurements. (a) Level diagram for the reset and readout protocols, where the letters $g, e$, $f$, and $h$ denote qubit energy levels and the numbers 0 and 1 denote resonator levels. Reset is performed by simultaneously driving both $|g 0\rangle \rightarrow|h 0\rangle$ and $|h 0\rangle \rightarrow|e 1\rangle$ transitions (blue double-headed arrows). The spontaneous photon decay $|e 1\rangle \rightarrow$ $|e 0\rangle$ provides a directional transition (blue single-headed arrow), removing the entropy and completing the reset. An $|e 0\rangle \rightarrow|f 0\rangle \pi$ pulse is applied before the readout to boost the output signal. (b) Rabi oscillations between $|e\rangle$ and $|f\rangle$ for different initial state preparations. Black circles: The initial state is the thermal equilibrium state. Blue squares: The initial state is prepared in $|e\rangle$ before the $|e\rangle \leftrightarrow|f\rangle$ Rabi. Orange diamonds: The initial state is prepared in $|g\rangle$.
$190 \mu \mathrm{K}$, lower than the ambient temperature by a factor of 100 .

Readout of the fluxonium levels is performed using circuit QED [30] by capacitively coupling the fluxonium circuit to a readout resonator [31]. Since the qubit states are far away in frequency from the readout resonator, the dispersive shift $\chi$ of the resonator due to a change in the occupation of computational states is small $(60 \mathrm{kHz})$. Therefore, while the large detuning reduces the qubit heating through the resonator, it makes direct dispersive readout challenging. We circumvent this issue by utilizing the larger dispersive interactions $\chi_{f}$ and $\chi_{h}$ of the excited levels $|f\rangle$ and $|h\rangle$, respectively, which are closer in frequency to the readout resonator. We thus perform a $\pi$ pulse on the $|e\rangle-|f\rangle$ transition in $80 \mathrm{~ns}$, before standard dispersive readout. Since the population in $|e\rangle$ is transferred to $|f\rangle$, the readout signal becomes proportional to $\left(\chi_{f}-\chi_{g}\right)$, which is 5 times larger than $\left(\chi_{e}-\chi_{g}\right)$. A previously reported dispersive shift engineering technique [28] for fluxoniums tries to increase $\left(\chi_{e}-\chi_{g}\right)$, while our protocol uses state selective transfer and the dispersive shift between the excited plasmon states and the computational states for readout. This plasmon-assisted readout scheme results in $50 \%$ single-shot readout fidelity, which can be further improved with a parametric amplifier and by optimizing the resonator $\kappa$ and the dispersive shifts (see Appendix F).

\section{CHARACTERIZING DEVICE COHERENCE}

Having developed protocols for initialization and readout, we characterize the coherence properties of the qubit. Figure 3(a) shows the experimentally measured $T_{1}$ (black circles) as a function of the applied external flux, while the inset shows $T_{1}=315 \pm 10 \mu \mathrm{s}$ measured at the fluxfrustration point $(\star)$ following initialization of the qubit in either the $|g\rangle$ or the $|e\rangle$ state. The qubit relaxes to a near-equal mixture where the excited state population $P(|e\rangle)=0.4955 \pm 0.0015$, with the deviation providing an estimate of the temperature of the surrounding bath, $T=42 \pm 14 \mathrm{mK}$. At the flux-frustration point, the wave functions are delocalized into symmetric and antisymmetric combinations of the states in each well. As we move away from this degeneracy point, the wave functions localize into different wells, resulting in a suppression of tunneling and an increase in the relaxation times; see Fig. 3(a). Here, the qubit relaxation times are measured over a wide range of external flux by driving the $|g\rangle-|h\rangle$ transition for $120 \mu \mathrm{s}$ (5 times the $|h\rangle$ state $T_{1}$ ) to ensure most of the population reside in the $|e\rangle$ state and monitoring the subsequent decay. While moving away from the flux-frustration point, $T_{1}$ increases to a maximum value of $4.3 \pm 0.2 \mathrm{~ms}$, consistent with previous heavy-fluxonium devices $[13,26]$, before subsequently decreasing.

To explain the measured relaxation times, we consider several avenues by which the qubit can decay, including 

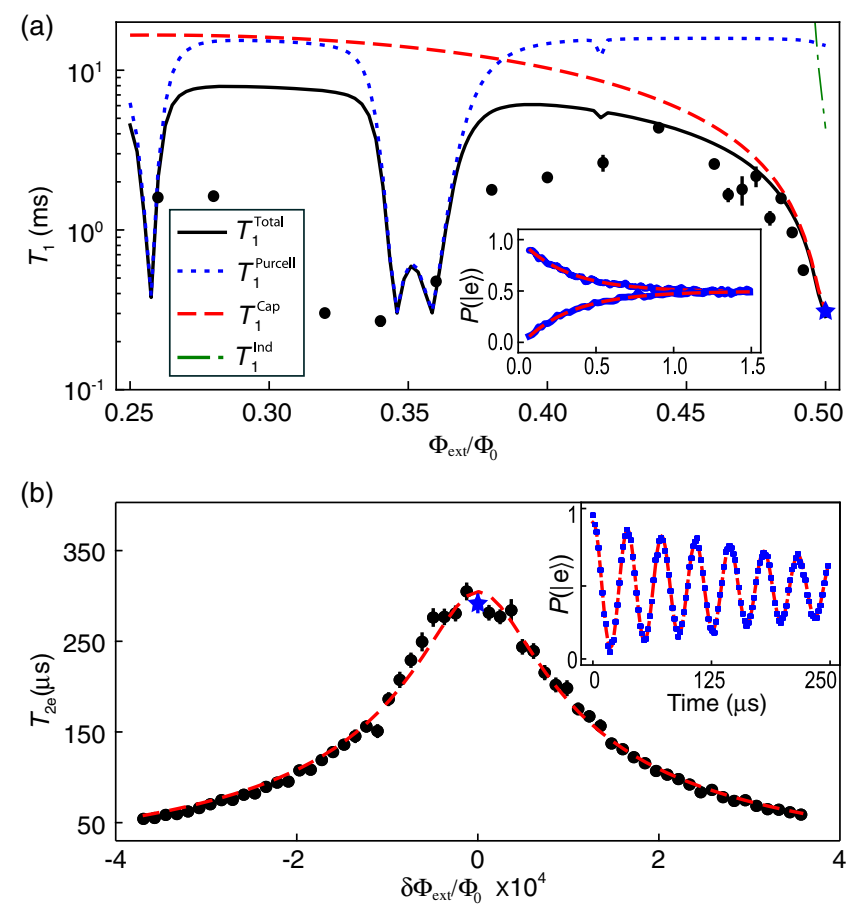

FIG. 3. Qubit coherence as a function of the flux. (a) Energy relaxation time $\left(T_{1}\right)$ as a function of the external flux, denoted by black circles. The lines represent theoretical limits set by dielectric $\left(T_{1}^{\text {cap }}\right)$, inductive $\left(T_{1}^{\text {ind }}\right)$, Purcell $\left(T_{1}^{\text {Purcell }}\right)$, and the combined loss $\left(T_{1}^{\text {total }}\right)$. The inset shows the decay of $P(|e\rangle)$ to 0.495 after preparing the qubit in $|g\rangle,|e\rangle$ at the flux-frustration point ( $\star$ ). (b) Echo decay time $T_{2 e}$ as a function of the flux near the flux-frustration point. The inset shows an echo measurement at the flux-frustration point $(\star)$.

Purcell loss, decay via charge and flux coupling to the control lines, $1 / f$ flux noise, dielectric loss in the capacitor, and resistive loss in the superinductor. Conservative estimates of the flux-noise-induced loss are lower than the measured loss by nearly an order of magnitude (see Appendix G). The loss near the flux-frustration point is believed to be largely due to dielectric loss in the capacitor. This loss can be thought of as Johnson-Nyquist current noise from the resistive part of the shunting capacitor, which couples to the phase matrix element $\langle g|\hat{\varphi}| e\rangle$ and grows rapidly as we approach the flux-frustration point [26]. Assuming a fixed loss tangent for the capacitor, this loss rate is inversely proportional to the impedance of the capacitor and is given by

$$
\Gamma_{\text {diel }}=\frac{\hbar \omega_{q}^{2}}{4 E_{C} Q_{\text {cap }}} \operatorname{coth}\left(\frac{\hbar \omega_{q}}{2 k_{B} T}\right)|\langle g|\hat{\phi}| e\rangle|^{2} .
$$

The $T_{1}$ at the flux-frustration point sets an upper bound of $1 / Q_{\text {cap }}=4 \times 10^{-6}$ for the loss tangent of the capacitor, which is within a factor of 2 of the value reported in previous heavy-fluxonium devices [26], and results in the dashed red curve in Fig. 3(a). Since $\omega_{q}$ is below the ambient temperature near the flux-frustration point, a combination of the temperature-dependent prefactor $\sim 2 k_{B} T /\left(\hbar \omega_{q}\right)$ and the relation between charge and phase matrix elements in fluxonium, $\langle g|\hat{n}| e\rangle=\omega_{q} /\left(8 E_{c}\right)\langle g|\hat{\phi}| e\rangle$, results in the dielectric-loss scaling as $1 / \omega_{q}$, which is consistent with the observed trend in the $T_{1}$ near the flux-frustration point. The measured $T_{1}$ at the flux-frustration point also sets an upper bound of $1.7 \times 10^{-9}$ for the loss tangent of the inductor. The decay from inductive loss, however, increases more rapidly with frequency than dielectric loss $\left(\propto 1 / \omega_{q}^{3}\right)$ and is inconsistent with measured data. Our qubit operations are performed between $0.4 \Phi_{0}$ and $0.5 \Phi_{0}$, where the $T_{1}$ is mainly limited by dielectric loss. As we move further away from the flux-frustration point (approximately $\left.0.4 \Phi_{0}\right), T_{1}$ starts to decrease. This additional loss is believed to be due to a combination of radiative loss to the charge drive line and Purcell loss from higher fluxonium levels excited by heating from the $|g\rangle$ and $|e\rangle$ states. The Purcell loss calculated based on the coupled fluxonium-resonator system using a bath temperature of $60 \mathrm{mK}$ results in the dotted blue curve shown in Fig. 3(a).

This result is slightly higher than the temperature inferred from the qubit thermal steady state population and can be attributed to the fact that the noise environments could be significantly different at frequencies that are more than 2 orders of magnitude apart. The enhanced loss near $\Phi_{\text {ext }}=$ $0.35 \Phi_{0}$ is suggestive that heating to higher levels may contribute, as there are several near resonances of higher fluxonium levels with the readout resonator, which depend sensitively on the circuit parameters (see Appendix G).

The dephasing is characterized using a Ramsey sequence with three echo $\pi$ pulses and found to be minimized at $\Phi_{\text {ext }}=\Phi_{0} / 2$, where the qubit frequency is first-order insensitive to changes in flux. The dephasing rate near the flux-frustration point can be separated into two parts. The first is a frequency-independent term $\Gamma_{C}$ mainly composed of qubit depolarization and dephasing from cavity photon shot noise and other flux-insensitive white noise sources. The second arises from $1 / f$ flux noise that is proportional to the flux slope as $\Gamma_{1 / f}=\left(d \omega / d \Phi_{\text {ext }}\right) \eta \sqrt{W}$, where $\eta$ is in the flux-noise amplitude and $W$ depends on the number of $\pi$ pulses in an echo experiment ( $W=4 \ln 2-\frac{9}{4} \ln 3$ for three $\pi$ pulses [32]). Thus, our spin-echo signal decays as $\exp \left(-t / T_{C}\right) \times \exp \left(-\Gamma_{1 / f}^{2} t^{2}\right)$. Here, $T_{C}=1 / \Gamma_{C}$ is the $T_{2 e}$ value at the flux-frustration point. It is found to be approximately $300 \mu \mathrm{s}$, much higher than the $T_{2 e}$ values for state-of-the-art transmons; see the inset in Fig. 3(b). The $T_{2}^{*}$ is found to be approximately 70-100 $\mu$ s (Fig. 9 in Appendix H), indicating that the dephasing is limited by low-frequency flux noise (see Appendix $\mathrm{H}$ ). The $T_{2 e}$ values around the flux-frustration point, defined as the time for the echo oscillation amplitude to decay to $1 / e$, are shown in Fig. 3(b). This value falls off rapidly as we move away from the flux-frustration point, 
consistent with the small tunnel coupling between levels. Away from the flux-frustration point, $T_{2 e}$ is mainly limited by $1 / f$ flux noise. The $T_{2 e}$ far from the frustration point is projected to be approximately $10 \mu \mathrm{s}$ according to our model, which is consistent with other reported results [26].

\section{FAST SINGLE-CYCLE FLUX GATES}

In order to maximize the advantage of the large anharmonicity of the heavy fluxonium, we rethink the standard microwave-drive control of the circuit which is hindered by the suppressed charge matrix elements. We instead control the qubit through fast-flux pulses and report the first use of nonadiabatic Landau-Zener transitions to realize ultrafast gates that occur within a single Larmor period. Near the flux-frustration point where the fluxonium is operated, the Hamiltonian within the computational space can be idealized as a spin-1/2 system, $(H / h)=$ $\left[A\left(\Phi_{\text {ext }}\right) / 2\right] \sigma_{x}+(\Delta / 2) \sigma_{z}$. Here, $\Delta \approx 14 \mathrm{MHz}$ is the splitting of $|g\rangle$ and $|e\rangle$ at the flux-frustration point and corresponds to the qubit frequency. The amplitude of the $\sigma_{x}$ term is proportional to the flux offset $\delta \Phi_{\text {ext }}$ from the flux-frustration point and given by $A=$ $4 \pi\langle g|\hat{\varphi}| e\rangle\left(E_{L} / h\right)\left(\delta \Phi_{\mathrm{ext}} / \Phi_{0}\right)$. The coefficient of the $\sigma_{x}$ term can be much larger than the qubit frequency, with $A \sim 300 \mathrm{MHz}$ when $\delta \Phi_{\text {ext }}=0.06 \Phi_{0}$, disallowing any rotating wave approximation.

Figure 4(a) shows the protocol for a generic qubit pulse. We first rapidly move the flux-bias point away from the flux-frustration point in one direction and back, thus generating a rotation about the $x$ axis through a large $\sigma_{x}$ term in our computational basis. There is additionally a relatively small rotation about the $z$ axis corresponding to the time $\Delta t_{p}$ of the triangular spike. We subsequently idle at the flux-frustration point for a duration $\Delta t_{z}$, which results in a rotation by $\omega_{q} \Delta t_{z}$ about the $z$ axis. Finally, we rapidly move the flux-bias point in the other direction and back, resulting in a $-\sigma_{x}$ term and another small $z$ rotation. We choose the two spikes to be exactly antisymmetric, ensuring zero net flux, simultaneously minimizing the effect of microsecond and millisecond pulse distortions ubiquitous in flux-bias lines [33], and echoing out low-frequency noise. The pulse is also immune to shape distortions, since the total $\sigma_{x}$ and $\sigma_{z}$ amplitudes depend only on the area of the spike and $\Delta t_{z}$. By sweeping the amplitude $A$ of the triangular spike and idling length $\Delta t_{z}$ of the pulse and measuring the expectation value of the spin along each axis, we obtain the 2D Rabi patterns shown in Fig. 4(c) that provide a measure of our gate parameters. A vertical line cut of these graphs corresponds to Larmor precession in the lab frame, with an oscillation frequency of $\Delta=14 \mathrm{MHz}$. We thus obtain a $Z / 2$ gate by idling at the flux-frustration point for $\Delta t_{z}=1 /(4 \Delta)$. We obtain a $Y / 2$ gate at the point indicated by the red star, with the corresponding trajectories on the Bloch sphere for three different cardinal states shown in Fig. 4(d). $Y / 2$ and arbitrary rotations about the (a)

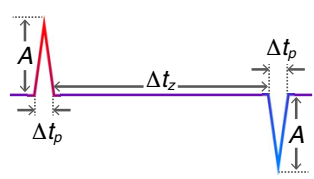

(b)
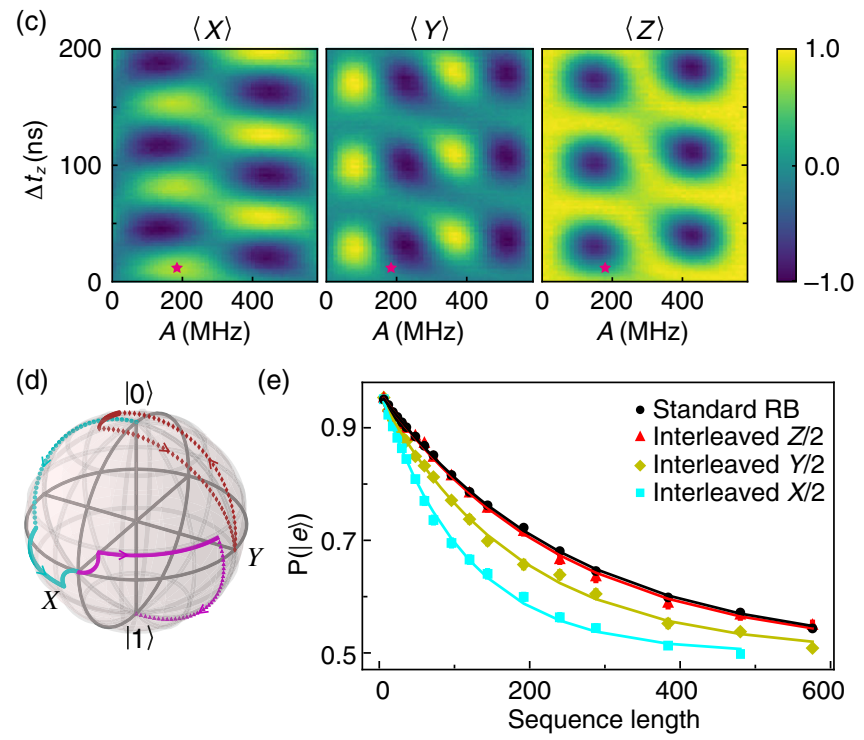

FIG. 4. Generic pulse scheme, gate calibration, and performance. (a) We use net-zero flux pulses as building blocks for universal control pulses. They are constructed using three sections, a positive triangular pulse with amplitude $A$ and width $\Delta t_{p}$ on the fast-flux line, an idling period of $\Delta t_{z}$, and, finally, another triangular pulse identical to the first one but with a negative amplitude. (b) Energy levels of the computational space as a function of the external flux $\left(\Phi_{\text {ext }}\right)$ showing how the fast-flux pulse changes the energies of the instantaneous eigenstates. (c) Expectation values of $\sigma_{x}, \sigma_{y}$, and $\sigma_{z}$ as a function of pulse parameters $\Delta t_{z}$ and $A$. These $2 \mathrm{D}$ sweeps are used to determine the optimal parameters for the $Y / 2$ and arbitrary $Z$ gates. $\star$ indicates the parameters for a $Y / 2$ gate. (d) Trajectories of three distinct initial states $|0\rangle$ (cyan), $(|0\rangle+|1\rangle) / \sqrt{2}$ (magenta), and $(|0\rangle+$ $i|1\rangle) / \sqrt{2}$ (brown) on the Bloch sphere when a $Y / 2$ gate is applied. (e) Comparison of standard RB (black circles) and interleaved $\mathrm{RB}$ for $Z / 2$ (red triangles), $Y / 2$ (gold diamonds), and $X / 2$ (cyan squares) gates. The plot is a result of 75 randomized gate sequences averaged over 10000 times. The average gate fidelity is $\mathcal{F}_{\text {avg }}=0.9980$, and the individual gate fidelities are $\mathcal{F}_{Z / 2}=0.9999, \mathcal{F}_{Y / 2}=0.9992$, and $\mathcal{F}_{X / 2}=0.9976$ [34]. The uncertainties in all fidelities are smaller than the least significant digit.

$z$ axis are sufficient for generating any single-qubit unitary, thus realizing universal control. An $X / 2$ gate, for instance, is performed through the combination $(-Y / 2) \cdot(Z / 2) \cdot(Y / 2)$.

We characterize the fidelities of our single-qubit gates through randomized benchmarking (RB) $[35,36]$ and interleaved RB (IRB) [34]. RB provides a measure of the average fidelity of single-qubit Clifford gates and is performed by applying sequences containing varying numbers of Clifford gates on the state $|e\rangle$. For a given 
sequence length, we perform 75 randomized sequences, each containing a recovery gate to the state $|e\rangle$ before the final measurement. IRB allows us to isolate the fidelities of individual computational gates and is performed by interleaving the gate between the random Clifford gates of the $\mathrm{RB}$ sequence. The averaged decay curves of $P(|e\rangle)$ as a function of the sequence length for standard $R B$ (black circles) and IRB for $Z / 2$ (red triangles), $Y / 2$ (gold diamonds), and $X / 2$ (cyan squares) gates are shown in Fig. 4(e). The infidelities thus extracted for the $Y / 2, Z / 2$, and $X / 2$ gates are 8,1 , and $24 \times 10^{-4}$, respectively. The $X / 2$ gate infidelity is slightly worse than the combined infidelities from two $Y / 2$ and one $Z / 2$ gate. The durations for $Y / 2$ and $Z / 2$ are approximately $20 \mathrm{~ns}$, while that for the $X / 2$ gate is approximately $60 \mathrm{ns,}$ and, thus, all the computational gates are performed within one qubit Larmor period $2 \pi / \omega_{q}=70 \mathrm{~ns}$ (see Appendix D), with all the operations occurring in the lab frame. The calculated decoherence limited errors of the $Y / 2$ and $X / 2$ gates are $6.67 \times 10^{-5}$ and $2 \times 10^{-4}$, respectively, suggesting that the major source of gate error arises from residual calibration errors in the pulse parameters, providing room for improvement even from these state-of-the-art values.

\section{CONCLUSION}

In conclusion, we have realized a heavy-fluxonium qubit with a $14 \mathrm{MHz}$ transition frequency and coherence times exceeding those of state-of-the-art transmons while demonstrating protocols for plasmon-assisted reset and readout of the qubit and a new flux control scheme that performs fast high-fidelity gates. We have explored a new frequency regime in superconducting qubits and demonstrated the feasibility of a subthermal frequency qubit, providing a path for manipulating fluxonium qubits with computational frequencies in the range of several gigahertz at temperatures much higher than current dilution-refrigerator temperatures. We have demonstrated improved coherence times over previous flux qubits and fluxoniums while using conventional fabrication processes that can be further improved by using new materials [37] or surface treatments [26]. Our control scheme has dramatically improved the single-qubit gate speed of fluxonium qubits, making them a viable candidate for large-scale superconducting quantum computation. The gate pulses can be directly synthesized with inexpensive digital-to-analog converters and are insensitive to shape distortions. Furthermore, they are broadly applicable to any system which can be driven nonadiabatically, including other low-frequency qubits, protected qubits, and interactions between qubits.

Our device is compatible with existing schemes for realizing two-qubit gates via capacitive coupling and the use of excited circuit levels [38]. The single-qubit gate scheme used in this work can also be generalized to two fluxonium circuits with strong inductive coupling, even comparable to the qubit frequencies. This generalization would allow for two-qubit gate operations that are as fast as the single-qubit gates demonstrated in this work, without involving the participation of the more lossy excited levels. Despite minimal optimization, we have realized a qubit with state-of-the-art gate fidelities and one of the longest reported coherence times in superconducting circuits. The readout and reset can also be improved by leveraging methods that have been demonstrated in other devices $[39,40]$. With the coherence times and gate speeds expected only to improve, the heavy-fluxonium circuit offers tantalizing prospects for scaling to processors of increased size and complexity.

\section{ACKNOWLEDGMENTS}

The authors thank Andrew Oriani for experimental assistance and Alex Ma, Brendan Saxberg, Alexander Anferov, and Jay Lawrence for useful discussions. This work was supported by the Army Research Office under Grant No. W911NF1910016. This work was partially supported by the University of Chicago Materials Research Science and Engineering Center, which is funded by the National Science Foundation under Grant No. DMR1420709. Devices were fabricated in the Pritzker Nanofabrication Facility at the University of Chicago, which receives support from Soft and Hybrid Nanotechnology Experimental (SHyNE) Resource (NSF ECCS-1542205), a node of the National Science Foundations National Nanotechnology Coordinated Infrastructure.

\section{APPENDIX A: EXPERIMENTAL SETUP}

The experiment is performed in a Bluefors LD-250 dilution refrigerator with the wiring configured as shown in Fig. 5. The flux and charge inputs are attenuated at the $4 \mathrm{~K}$ stage and the mixing chamber with standard XMA attenuators, except the final $20 \mathrm{~dB}$ attenuator on the rf charge line (threaded copper). The dc and rf-flux signals are combined in a modified bias tee (Mini-Circuits ${ }^{\circledR}$ ZFBT$4 \mathrm{R} 2 \mathrm{GW}+$ ), with the capacitor replaced with a short. The dc and rf-flux lines included commercial low-pass filters (Mini-Circuits ${ }^{\circledR}$ ) as indicated. The rf flux and output lines also have additional low-pass filters with a sharp cutoff (8 GHz) from K\&L microwave. Eccosorb (CR110) IR filters are added on the flux and output lines, which helps improve the $T_{1}$ and $T_{2}$ times and reduces the qubit and resonator temperatures. The device is heat sunk to the base stage of the refrigerator (stabilized at $15 \mathrm{mK}$ ) via an oxygen-free high-conductivity copper post while surrounded by an inner lead shield thermalized via a welded copper ring. This shield is additionally surrounded by two cylindrical $\mu$-metal cans (MuShield), thermally anchored using an inner close-fit copper shim sheet, attached to the copper can lid. We ensure that the sample shield is light tight, to reduce thermal photons from the environment. 


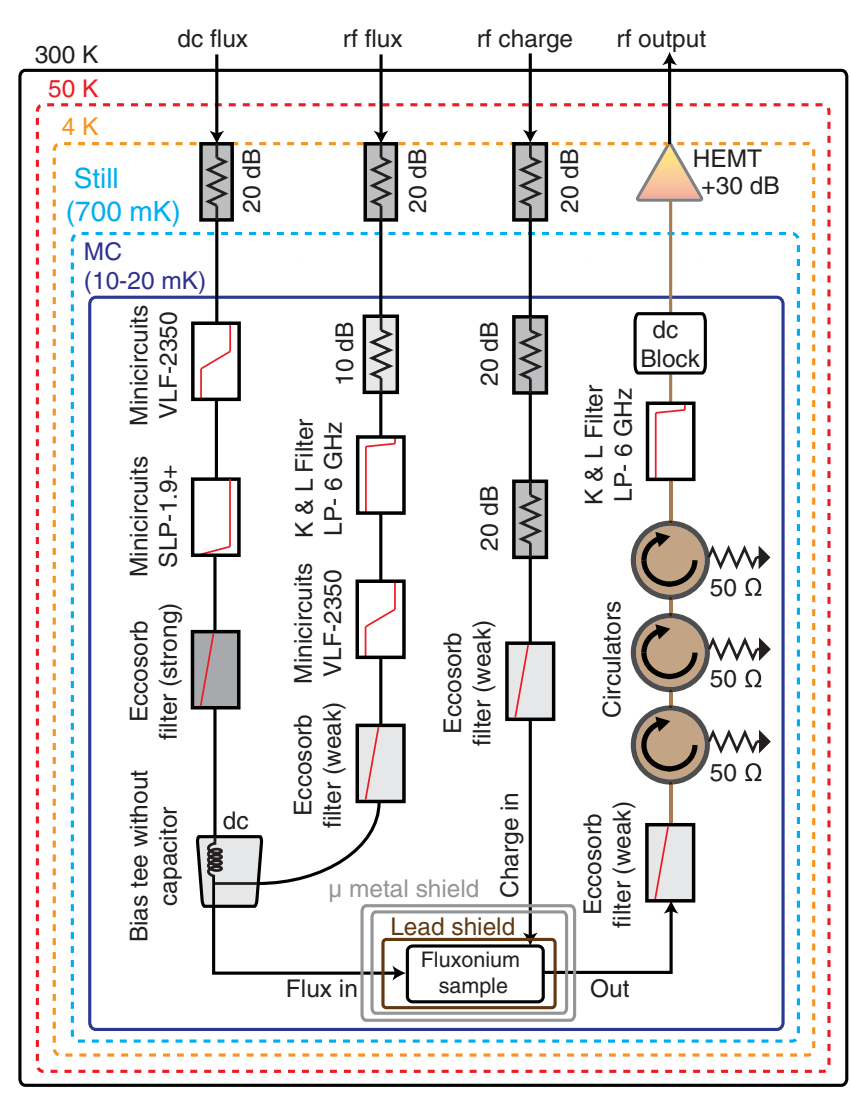

FIG. 5. Wiring diagram inside the dilution refrigerator. Outside the dilution fridge, there is approximately $16 \mathrm{~dB}$ of attenuation and a dc block on the rf-flux line and an ultralow-pass (approximately $1 \mathrm{~Hz}$ ) $R C$ filter on the dc flux line. The total attenuation on the rf-flux line proves important for both the $T_{1}$ and $T_{2}$ of the qubit, likely due to reduction in noise from the arbitrary waveform generator (Agilent $81180 \mathrm{~A}$ ).

\section{APPENDIX B: DEVICE FABRICATION}

The device (shown in Fig. 1 in the main text) is fabricated on a $430-\mu \mathrm{m}$-thick C-plane sapphire substrate. The base layer of the device, which includes the majority of the circuit (excluding the Josephson junctions), consists of $150 \mathrm{~nm}$ of niobium deposited via electron-beam evaporation, with features fabricated via optical lithography and reactive ion etch (RIE) at wafer scale. A 600-nm-thick layer of AZ MiR 703 is used as the (positive) photoresist, and the large features are written using a Heidelberg MLA 150 Direct Writer, followed by RIE performed using a PlasmaTherm ICP fluorine etch tool. The junction mask is fabricated via electron-beam lithography with a bilayer resist (MMA-PMMA) comprising of MMA EL11 and 950PMMA A7. The $e$-beam lithography is performed on a Raith EBPG5000 Plus E-Beam Writer. All Josephson junctions are made with the Dolan bridge technique. They are subsequently evaporated in a Plassys electron beam evaporator with double angle evaporation $\left( \pm 19^{\circ}\right)$. The wafer is then diced into $7 \times 7 \mathrm{~mm}$ chips, mounted on a printed circuit board, and subsequently wire bonded.

\section{APPENDIX C: DECONSTRUCTION OF SINGLE-QUBIT GATES}

Modulation of the external flux drive with appropriate amplitude and duration is sufficient to perform arbitrary single-qubit rotations. The native gates available in our system are the arbitrary phase gate $R_{z}(\theta)$, which rotates the qubit by an arbitrary angle $\theta$ about the $Z$ axis, and a combination of $X$ and $Z$ rotation $R_{x z}(\theta) . R_{z}(\theta)$ is realized by waiting for a period of $\Delta t_{z}=\theta / \omega_{q}$ (since we are working in the lab frame), whereas $R_{x z}(\theta)$ is implemented by a flux drive applied for a duration of $\Delta t_{p}=\lambda \theta / \omega_{q}$. Here, $\lambda(\lambda \leq 1)$ is the ratio of $Z$ rotation to $X$ rotation rates. These rotation matrices can be expressed, respectively, as

$$
\begin{gathered}
R_{z}(\theta)=e^{-i \sigma_{z} \theta / 2}, \\
R_{x z}(\theta)=e^{-i\left(\theta \sigma_{x}+\lambda|\theta| \sigma_{z}\right) / 2} .
\end{gathered}
$$

The $|\theta|$ in Eq. (C2) arises due to the always-on $Z$ rotation, which is unidirectional in the lab frame. A generic zero-flux pulse can be constructed as

$$
R(\theta)=R_{x z}\left(-\theta_{x}\right) \cdot R_{z}\left(\theta_{z}\right) \cdot R_{x z}\left(\theta_{x}\right) .
$$

A $\pi / 2$ rotation about the $Y$ axis $(Y / 2)$, i.e.,

$$
R_{y}(\pi / 2)=\frac{1}{\sqrt{2}}\left(\begin{array}{cc}
1 & -1 \\
1 & 1
\end{array}\right)
$$

is obtained using

$$
\begin{gathered}
\theta_{x}=\frac{1}{\sqrt{1+\lambda^{2}}} \cos ^{-1}\left[\frac{\lambda(1+\lambda)}{-(1-\lambda)}\right], \\
\theta_{z}=2 \tan ^{-1}\left[\frac{\sqrt{1-2 \lambda-2 \lambda^{3}-\lambda^{4}}}{(1+\lambda) \sqrt{1+\lambda^{2}}}\right]
\end{gathered}
$$

in Eq. (C3) provided $0 \leq \lambda \leq \sqrt{2}-1$. Similarly, we can construct

$$
R_{y}(\pi)=\left(\begin{array}{cc}
0 & -1 \\
1 & 0
\end{array}\right)=-i \sigma_{y}
$$

using

$$
\begin{gathered}
\theta_{x}=\frac{1}{\sqrt{1+\lambda^{2}}} \cos ^{-1}\left(\lambda^{2}\right), \\
\theta_{z}=\pi-2 \tan ^{-1}\left[\frac{\lambda}{\sqrt{1-\lambda^{2}}}\right],
\end{gathered}
$$

with $0 \leq \lambda \leq 1$. An arbitrary rotation about the $X$ axis can be constructed using 


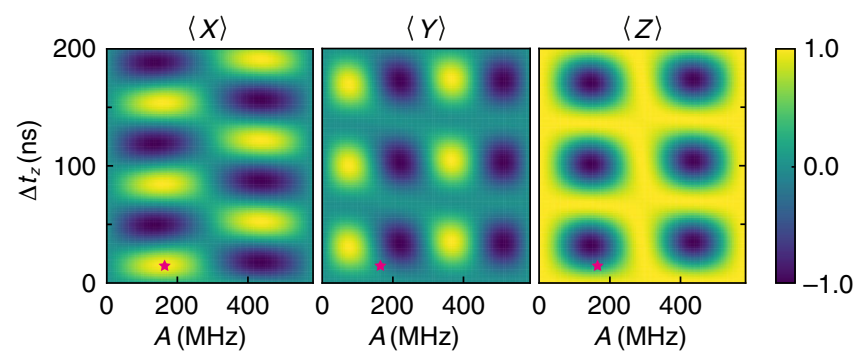

FIG. 6. Simulated expectation values of $\sigma_{x}, \sigma_{y}$, and $\sigma_{z}$ as a function of pulse parameters $\Delta t_{z}$ and $A$ with $\Delta t_{p}=4.76 \mathrm{~ns}$. The simulation shows extremely good agreement with the experimental data as shown in Fig. 4(c).

$$
R_{x}(\theta)=R_{y}(\pi / 2) \cdot R_{z}(\theta) \cdot R_{y}(-\pi / 2)
$$

These gates are sufficient to construct any single-qubit unitary operation. We use the QuTiP [41] PYTHON package to simulate the evolution of the computational levels under application of the pulse that is shown in Fig. 3 and obtain the gate parameters. We sweep the drive amplitude $A$ and idling period $\Delta t_{z}$ in our simulation to match the sweep performed in the experiment, as shown in Fig. 6. $\Delta t_{p}=$ $4.76 \mathrm{~ns}$ in all the experiments and simulations reported in this paper.

\section{APPENDIX D: CLIFFORD GATE LENGTHS AND FIDELITIES}

A complete Clifford set includes the computational gates $\left[\exp \left( \pm i \pi \sigma_{j} / 4\right), j=x, y\right]$ and the Pauli gates $\left[\exp \left( \pm i \pi \sigma_{j} / 2\right), j=I, x, y, z\right]$. In this work, we construct $Y / 2$ and $Z / 2$ gates and use them as building blocks for the other gates in the Clifford set. The total gate lengths, experimental infidelities (computational gates only), and gate compositions are shown in Table I. The computational gate lengths range from 21 to $60 \mathrm{~ns}$, and the longest Pauli gate $(X)$ has a length of $78 \mathrm{~ns}$. Since $2 \pi / \omega_{q} \approx 70 \mathrm{~ns}$, the computational gates are all within a single cycle of the qubit, and the longest gate is around one cycle as well. These single-cycle flux gates are 10-30 times faster than standard microwave-driven gates, whose durations are longer than $10 \times 2 \pi / \omega_{q}$.

TABLE I. Clifford gates.

\begin{tabular}{lccc}
\hline \hline Gate & Length (ns) & Exp. infidelity & Gate composition \\
\hline$Y / 2$ & 21.19 & $8 \times 10^{-4}$ & \\
$Z / 2$ & 17.87 & $1 \times 10^{-4}$ & \\
$X / 2$ & 60.25 & $24 \times 10^{-4}$ & $Y / 2, Z / 2,-Y / 2$ \\
$Y$ & 42.38 & & $Y / 2, Y / 2$ \\
$Z$ & 35.73 & & $Z / 2, Z / 2$ \\
$X$ & 78.11 & & $Y / 2, Z,-Y / 2$ \\
\hline \hline
\end{tabular}

\section{APPENDIX E: FLUXONIUM MATRIX ELEMENTS AND RESET PROTOCOL}

We derive the charge drive transition rates by simulating the full qubit-resonator dressed system. The drive power is normalized to $258 \mathrm{MHz}$ so that the $|g 0\rangle \rightarrow|h 0\rangle \pi$ pulse takes $80 \mathrm{~ns}$, which corresponds to the typical experimental value. The simulated single-photon and two-photon transition rates (in megahertz) are shown in Tables II and III. The observed transition rates have additional contributions arising from the frequency dependence of the transmission through the drive line.

We utilize the $|g 0\rangle \rightarrow|h 0\rangle$ and $|h 0\rangle \rightarrow|e 1\rangle$ transitions for the reset protocol due to their large matrix elements. In principle, any transition combination that leads to fast, unidirectional population transfer can be used as the reset transitions. We choose the protocol based on the strength of the coupling between different states and the detailed level structure for fastest reset. The excited state population as a function of the reset time is shown in Fig. 7. The majority of the population is pumped to state $|e\rangle$ in $5 \mu \mathrm{s}$, which is mainly determined by the $|h 0\rangle \rightarrow|e 1\rangle$ transition rate. The reset process continues for another $10 \mu$ s until the steady state $(97 \%$ in $|e\rangle)$ is reached. We subsequently perform an additional $\pi$ pulse on the $|g\rangle-|e\rangle$ transition to initialize the system in the ground state $|g 0\rangle$.

\section{APPENDIX F: PLASMON-ASSISTED READOUT}

The resonator frequency shifts in increasing order are $\chi_{e}$, $\chi_{g}, \chi_{h}$, and $\chi_{f}$. We select the $|g\rangle$ and $|f\rangle$ states for plasmonassisted readout, since $\chi_{f}-\chi_{g}$ is larger than $\chi_{h}-\chi_{e}$. This result is reflected in the single-shot readout histogram data for $|g\rangle,|e\rangle,|f\rangle$, and $|h\rangle$ as shown in Fig. 8. The histograms

TABLE II. Single-photon matrix elements.

\begin{tabular}{|c|c|c|c|c|c|c|}
\hline & $|g 0\rangle$ & $|e 0\rangle$ & $|f 0\rangle$ & $|h 0\rangle$ & $|g 1\rangle$ & $|e 1\rangle$ \\
\hline$|g 0\rangle$ & & 0.0738 & & 6.2577 & 257.9425 & \\
\hline$|e 0\rangle$ & 0.0738 & & 5.8679 & & & 257.9108 \\
\hline$|f 0\rangle$ & & 5.8679 & & 1.2475 & 0.0138 & \\
\hline$|h 0\rangle$ & 6.2577 & & 1.2475 & & & 0.10 \\
\hline$|g 1\rangle$ & 257.9425 & & 0.0138 & & & 0.0741 \\
\hline$|e 1\rangle$ & & 257.9108 & & 0.1028 & 0.0741 & \\
\hline
\end{tabular}

TABLE III. Two-photon matrix elements $\times 10^{3}$.

\begin{tabular}{lcccccc}
\hline \hline & $|g 0\rangle$ & $|e 0\rangle$ & $|f 0\rangle$ & $|h 0\rangle$ & $|g 1\rangle$ & $|e 1\rangle$ \\
\hline$|g 0\rangle$ & & & 1.9213 & & & 0.9177 \\
$|e 0\rangle$ & & & & 1.6489 & 0.4207 & \\
$|f 0\rangle$ & 1.9213 & & & & & 0.0644 \\
$|h 0\rangle$ & & 1.6489 & & & 0.1258 & \\
$|g 1\rangle$ & & 0.4207 & & 0.1258 & & \\
$|e 1\rangle$ & 0.9177 & & 0.0644 & & & \\
\hline \hline
\end{tabular}




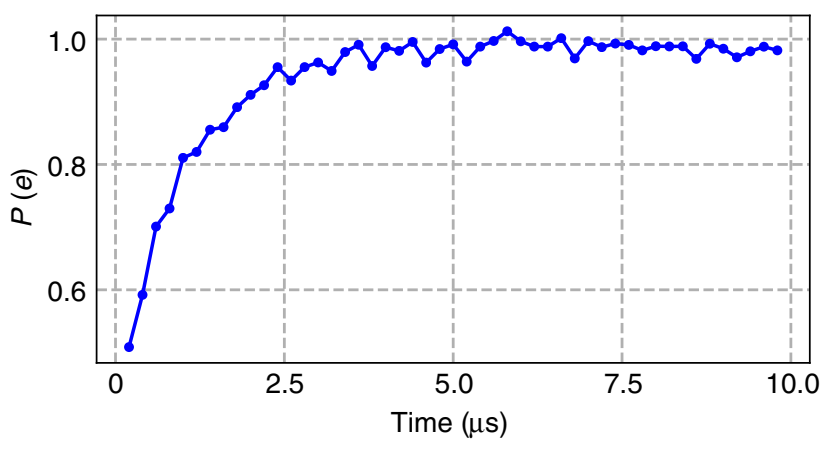

FIG. 7. The population in the $|e\rangle$ state as a function of the length of the reset pulse. The population is measured after simultaneously driving the $|g 0\rangle \rightarrow|h 0\rangle$ and $|h 0\rangle \rightarrow|e 1\rangle$ transitions for different lengths of time. Reset of the state is achieved in approximately $5 \mu \mathrm{s}$. (a)

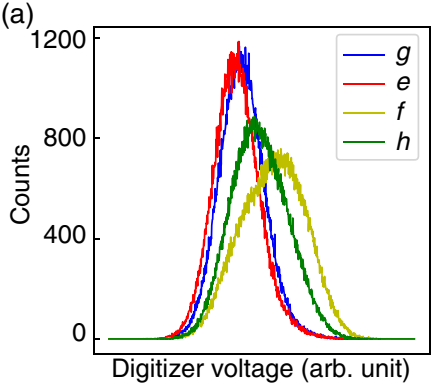

(b)

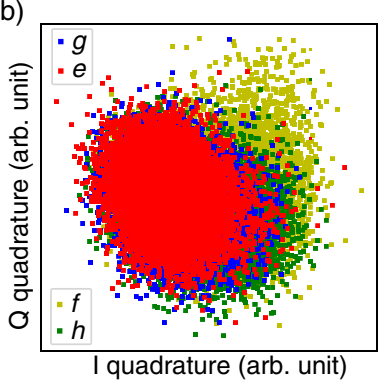

FIG. 8. Readout histogram and single-shot data (a) histogram of the lowest four fluxonium states $(|g\rangle,|e\rangle,|f\rangle$, and $|h\rangle)$. The $|g\rangle-|f\rangle$ readout fidelity is approximately $50 \%$. (b) The distribution of all single-shot data from the lowest four fluxonium states on the IQ (IQ are defined from the two IF channels of the I/ $\mathrm{Q}$ mixers. I for the in-phase component and Q for the $90^{\circ}$ out-ofphase component) plane.

are not well separated, since the current sample is not optimized for high-fidelity readout. There are several avenues for further increasing the readout fidelity. The dispersive shifts of the plasmon levels can be increased by engineering the device parameters to bring some excited circuit levels closer to those of the resonator. We can further optimize readout by exploring different flux bias points to take full advantage of the metastability of the fluxon. Lastly, the rich excited state spectrum allows for the use of different excited states for population transfer and dispersive shift engineering.

\section{APPENDIX G: MODELING FLUXONIUM RELAXATION}

To explain the measured relaxation times of the fluxonium, we consider decay via charge and flux coupling to the control lines, $1 / f$ flux noise, dielectric loss in the capacitor, resistive loss in the superinductor, and Purcell loss. The decay rates arising from these loss mechanisms are derived using Fermi's golden rule, with the bath described using the Caldeira-Leggett model $[42,43]$. For a noise source with amplitude $f(t)$ and coupling constant $\alpha$ between the fluxonium qubit states, the interaction Hamiltonian can be written as $H^{\prime}=\alpha f(t) \sigma_{x}$ in the qubit subspace. This derivation results in a qubit depolarization rate

$$
\Gamma=\frac{\alpha^{2}}{\hbar^{2}}\left[S_{f}\left(+\omega_{01}\right)+S_{f}\left(-\omega_{01}\right)\right] .
$$

Here, $S_{f}(\omega)=\int_{-\infty}^{\infty} e^{i \omega \tau}\langle f(\tau) f(0)\rangle$ is the noise spectral density associated with the source. We note that, at a finite bath temperature corresponding to an inverse temperature $\beta=\left(1 / k_{B} T\right)$, detailed balance relates the positive and negative frequency components of the noise spectral density as $S_{f}(-\omega) / S_{f}(\omega)=e^{-\beta \hbar \omega}$. Depending on the noise source $f$, the coupling constant $\alpha$ is proportional to the charge or phase matrix element of the fluxonium. Since the only term in the Hamiltonian that does not commute with $\hat{\phi}$ is the charging energy $4 E_{c} \hat{n}^{2}$, and $[\hat{\phi}, \hat{n}]=i$,

$\langle j|[\hat{\phi}, \hat{H}]| k\rangle=\left(\omega_{j}-\omega_{k}\right)\langle j|\hat{\phi}| k\rangle=i\left(8 E_{c}\right)\langle j|\hat{n}| k\rangle$.

The matrix elements of the fluxonium circuit are, thus, related by $|\langle g 0|\hat{n}| g 1\rangle|=\left(\omega / 8 E_{c}\right)|\langle g 0|\hat{\phi}| g 1\rangle|$ for all flux values.

\section{Relaxation from flux noise}

Flux noise couples to the phase degree of freedom with an interaction strength that depends on the inductive energy $E_{L}$. Expanding the fluxonium potential to lowest order in flux results in a coupling constant of $\alpha=$ $2 \pi E_{L}\langle g 0|\hat{\varphi}| g 1\rangle / \Phi_{0}$. We consider flux-noise contributions from current noise in the flux-bias line, as well as $1 / f$ flux noise. In our experimental setup, the current noise is believed to be mainly due to resistive Johnson-Nyquist noise arising from a $10 \mathrm{~dB}$ attenuator with resistance $R=$ $26 \Omega$ (last resistor in the $T$ network) on the fast-flux line, corresponding to a current noise spectral density of $S_{I}(\omega)=(2 / R)\left[\hbar \omega /\left(1-e^{-\beta \hbar \omega}\right)\right]$, with the expected interpolation between quantum and thermal noise. This noise is related to flux noise by the mutual inductance $M=$ $\Phi_{0} / 1.6 \mathrm{~mA}$ between the flux line and the qubit, obtained from the dc flux period. Therefore, $S_{f}(\omega)+S_{f}(-\omega)=$ $2 \hbar \omega\left(M^{2} / R\right) \operatorname{coth}(\beta \hbar \omega / 2)$, and the decay rate

$\Gamma_{R}=\pi^{3}\left(\frac{R_{Q}}{R}\right)\left(\frac{M}{L}\right)^{2}|\langle g 0|\hat{\varphi}| g 1\rangle|^{2} \omega \operatorname{coth}\left(\frac{\beta \hbar \omega}{2}\right)$,

where $R_{Q}=h / e^{2}$ is the resistance quantum and $L$ is the fluxonium inductance.

For $1 / f$ flux noise, the noise spectral density is of the form $S_{\Phi}(\omega)=2 \pi \eta^{2} / \omega$, with the resulting decay rate 


$$
\Gamma_{1 / f}=8 \pi^{3}\left(\frac{E_{L}}{\hbar}\right)^{2}\left(\frac{\eta}{\Phi_{0}}\right)^{2} \frac{|\langle g 0|\hat{\varphi}| g 1\rangle|^{2}}{\omega} .
$$

The $1 / f$ noise amplitude is fit from $T_{2 e}$ data and corresponds to $\eta=5.21 \mu \Phi_{0}$. The suppression of the $1 / f$ noiseinduced decay by $E_{L}^{2}$ results in a limit of $T_{1}=2.4 \mathrm{~ms}$ for the relaxation time at the flux-frustration point, which grows rapidly $\left(\propto \omega^{3}\right)$ as we move away from it.

\section{Relaxation from radiative loss to the charge line}

In addition to current noise, the fluxonium could also be affected by radiative loss arising from Johnson-Nyquist voltage noise $\left[S_{V}(\omega)=\left(2 R \hbar \omega / 1-e^{-\beta \hbar \omega}\right)\right]$ that couples to the qubit via spurious charge coupling, with the resistance $R$ serving as a phenomenological parameter. In this case, the coupling constant is related to the charge matrix element as $\alpha=2 e\langle g 0|\hat{n}| g 1\rangle$, and $S_{f}(\omega)+S_{f}(-\omega)=$ $2 R \hbar \omega \operatorname{coth}(\beta \hbar \omega / 2)$. The resulting decay rate is

$$
\Gamma_{c}=\frac{\omega}{Q_{c}} \operatorname{coth}\left(\frac{\beta \hbar \omega}{2}\right)|\langle g 0|\hat{n}| g 1\rangle|^{2},
$$

where $Q_{c}=\left(R_{Q} / 16 \pi R\right)$. An upper bound for the resistance $R$ can be found using the plasmon $T_{1}$ of $10 \mu \mathrm{s}$, corresponding to a total quality factor of $1.86 \times 10^{5}$, and $Q_{c}=7.4 \times 10^{4}$. This bound results in a fluxon $T_{1}$ limit in excess of $60 \mathrm{~ms}$ at the flux-frustration point.

\section{Relaxation from dielectric loss in the capacitor}

Dielectric loss associated with the capacitor can be thought of as Johnson-Nyquist current noise from the resistive part of the shunting capacitor, which couples to the phase matrix element $\langle g|\hat{\varphi}| e\rangle$. This loss rate is, therefore, inversely proportional to the impedance of the capacitor, assuming a fixed loss tangent $\left(1 / Q_{\text {diel }}\right)$ for the capacitor. As a result, $S_{f}(\omega)+S_{f}(-\omega)=$ $\left(2 \hbar \omega^{2} C / Q_{\text {diel }}\right) \operatorname{coth}(\beta \hbar \omega / 2)$, and

$$
\Gamma_{\text {diel }}=\frac{\hbar \omega^{2}}{4 E_{C} Q_{\text {cap }}} \operatorname{coth}\left(\frac{\beta \hbar \omega}{2}\right)|\langle g 0|\hat{\phi}| g 1\rangle|^{2} .
$$

If the $T_{1}$ at the frustration point is limited by dielectric loss, a bath temperature of $42 \mathrm{mK}$ results in $Q_{\text {cap }}=$ $1 /\left(4 \times 10^{-6}\right)$. This loss tangent is slightly larger but within a factor of 3 of that observed in similar fluxonium devices [26]. Dielectric loss is believed to be the dominant loss channel near the frustration point, also capturing the flux and frequency dependence of the measured loss $(\propto 1 / \omega)$.

\section{Relaxation from resistive loss in the inductor}

For inductive loss, we again assume a frequencyindependent loss tangent $\left[L \rightarrow L\left(1+i / Q_{\text {ind }}\right)\right]$, resulting in Johnson-Nyquist current noise that is inversely proportional to the impedance of the superinductor, i.e., $S_{f}(\omega)+S_{f}(-\omega)=\left(\hbar / L Q_{\text {ind }}\right) \operatorname{coth}(\beta \hbar \omega / 2)$. The inductive loss is, thus,

$$
\Gamma_{\text {ind }}=\frac{2 E_{L}}{\hbar Q_{L}} \operatorname{coth}\left(\frac{\beta \hbar \omega}{2}\right)|\langle g 0|\hat{\phi}| g 1\rangle|^{2} .
$$

The superinductor is extremely low loss, with a quality factor of $Q_{\text {ind }}=8 \times 10^{9}$ resulting in a limit of $T_{1}=4 \mathrm{~ms}$ at the flux-frustration point, growing as $\omega^{3}$ as we move away from the flux-frustration point.

\section{Relaxation rate due to the Purcell effect}

We derive the Purcell relaxation rates of the fluxonium levels, arising from coupling to the resonator by closely following Ref. [44]. We model this coupling by assuming that the resonator is coupled to a bath of harmonic oscillators, whose Hamiltonian reads

$$
H_{\text {bath }}=\sum_{k} \hbar \omega_{k} b_{k}^{\dagger} b_{k}
$$

where $b_{k}$ is the lowering operator for mode $k$. The interaction Hamiltonian between the bath and the resonator is given by

$$
H_{\mathrm{int}}=\hbar \sum_{k} \lambda_{k}\left(a b_{k}^{\dagger}+a^{\dagger} b_{k}\right)
$$

where $a$ is the lowering operator for the resonator. Finally, the system under consideration is the fluxonium circuit coupled to the resonator, which we write in the dressed basis as

$$
H_{\text {flux }+ \text { res }}=\sum_{k} E_{k}^{\text {flux }+ \text { res }}\left|\psi_{k}^{\text {flux }+ \text { res }}\right\rangle\left\langle\psi_{k}^{\text {flux }+ \text { res }}\right| .
$$

We treat $H_{\text {int }}$ as a perturbation which can induce transitions among the eigenstates of the Hamiltonian $H=$ $H_{\text {bath }}+H_{\text {flux }+ \text { res }}$, given by

$$
\left|\psi_{i}\right\rangle=\left|\psi_{i}^{\mathrm{flux}+\mathrm{res}}\right\rangle \underset{k}{\otimes}\left|m_{k}\right\rangle .
$$

The transition rate under the action of a constant perturbation is given by Fermi's golden rule in the form

$$
\gamma_{i \rightarrow f}=\frac{2 \pi}{\hbar} \delta\left(E_{i}-E_{f}\right)\left|\left\langle\psi_{f}\left|H_{\mathrm{int}}\right| \psi_{i}\right\rangle\right|^{2}
$$

where $E_{i}$ and $E_{f}$ are the eigenenergies of the states $\left|\psi_{i}\right\rangle$ and $\left|\psi_{f}\right\rangle$, respectively. These energies are 


$$
\begin{aligned}
& E_{i}=E_{i}^{\mathrm{flux}+\mathrm{res}}+\hbar \sum_{k} m_{k} \omega_{k}, \\
& E_{f}=E_{f}^{\mathrm{flux}+\mathrm{res}}+\hbar \sum_{k} m_{k}^{\prime} \omega_{k},
\end{aligned}
$$

where $\left\{m_{k}\right\}$ denotes the initial configuration of the bath and $\left\{m_{k}^{\prime}\right\}$ the final configuration. Inserting the form of $H_{\text {int }}$ into Eq. (G12) and noting that cross terms vanish leads to

$$
\begin{aligned}
\gamma_{i,\left\{m_{k}\right\} \rightarrow f,\left\{m_{k}^{\prime}\right\}}= & 2 \pi \hbar \delta\left(E_{i}-E_{f}\right) \sum_{k}\left|\lambda_{k}\right|^{2}\left[\left|\left\langle\psi_{f}^{\text {flux }+ \text { res }}\left|a^{\dagger}\right| \psi_{i}^{\text {flux }+ \text { res }} \mid\right\rangle\right|^{2} m_{k} \delta_{m_{k}^{\prime}, m_{k}-1}\right. \\
& \left.+\left|\left\langle\psi_{f}^{\text {flux }+ \text { res }}|a| \psi_{i}^{\text {flux }+ \text { res }}\right\rangle\right|^{2}\left(m_{k}+1\right) \delta_{m_{k}^{\prime}, m_{k}+1}\right] \prod_{k^{\prime} \neq k} \delta_{m_{k^{\prime}}, m_{k^{\prime}}} .
\end{aligned}
$$

To find the total transition rate, we must sum over all such initial and final configurations, taking into account the thermal probability of occupying a given initial configuration:

$$
\Gamma_{i \rightarrow f}=\sum_{\left\{m_{k}\right\},\left\{m_{k}^{\prime}\right\}} P\left(\left\{m_{k}\right\}\right) \gamma_{i,\left\{m_{k}\right\} \rightarrow f,\left\{m_{k}^{\prime}\right\}},
$$

where

$$
P\left(\left\{m_{k}\right\}\right)=\frac{e^{-\sum_{k} \beta m_{k} \hbar \omega_{k}}}{Z},
$$

$Z$ is the partition function of the bath, and $\beta=1 / k_{B} T$. Performing the sums over all initial and final states yields

$$
\begin{aligned}
\Gamma_{i \rightarrow f}= & 2 \pi \hbar \sum_{k}\left|\lambda_{k}\right|^{2} \delta\left(E_{i}^{\text {flux }+ \text { res }}-E_{f}^{\text {flux }+ \text { res }}+\hbar \omega_{k}\right) \\
& \left|\left\langle\psi_{f}^{\text {flux }+ \text { res }}\left|a^{\dagger}\right| \psi_{i}^{\text {flux }+ \text { res }}\right\rangle\right|^{2} n_{\text {th }}\left(\omega_{k}\right) \\
& +2 \pi \hbar \sum_{k}\left|\lambda_{k}\right|^{2} \delta\left(E_{i}^{\text {flux }+ \text { res }}-E_{f}^{\text {flux }+ \text { res }}-\hbar \omega_{k}\right) \\
& \left|\left\langle\psi_{f}^{\text {flux }+ \text { res }}|a| \psi_{i}^{\text {flux }+ \text { res }}\right\rangle\right|^{2}\left[n_{\text {th }}\left(\omega_{k}\right)+1\right],
\end{aligned}
$$

where

$$
n_{\mathrm{th}}\left(\omega_{j}\right)=\sum_{\left\{m_{k}\right\}} P\left(\left\{m_{k}\right\}\right) m_{j}=\frac{1}{e^{\beta \hbar \omega_{j}}-1} .
$$

We next take the continuum limit and define $\kappa=$ $2 \pi \hbar \rho\left(\omega_{k}\right)\left|\lambda_{k}\right|^{2}$, where $\rho(\omega)$ is the density of states of the bath. Introducing $\omega_{j j^{\prime}}^{\text {flux }+ \text { res }}=\left(E_{j}^{\text {flux }+ \text { res }}-E_{j^{\prime}}^{\text {flux }+ \text { res }}\right) / \hbar$ leads to the expressions

$$
\Gamma_{i \rightarrow f}^{\uparrow}=\kappa n_{\mathrm{th}}\left(\omega_{f i}^{\mathrm{flux}+\mathrm{res}}\right)\left|\left\langle\psi_{f}^{\text {flux }+ \text { res }}\left|a^{\dagger}\right| \psi_{i}^{\mathrm{flux}+\mathrm{res}}\right\rangle\right|^{2},
$$

for upward transitions $E_{f}^{\text {flux }+ \text { res }}>E_{i}^{\text {flux }+ \text { res }}$, and

$$
\Gamma_{i \rightarrow f}^{\downarrow}=\kappa\left[n_{\mathrm{th}}\left(-\omega_{f i}^{\text {flux }+ \text { res }}\right)+1\right]\left|\left\langle\psi_{f}^{\text {flux }+ \text { res }}|a| \psi_{i}^{\text {flux }+ \text { res }}\right\rangle\right|^{2},
$$

for downward transitions $E_{f}^{\text {flux }+ \text { res }}<E_{i}^{\text {flux }+ \text { res }}$. The final step is to note that, throughout this experiment, the fluxonium qubit is operated in the dispersive regime with respect to the frequency of the resonator. Therefore, we expect that the dressed eigenstates of $H_{\text {flux }+ \text { res }}$ can be labeled with quantum numbers $\ell$ and $n$, with $\ell$ labeling the fluxonium state and $n$ the resonator state. When performing numerical simulations, this identification is based on which numbers $\ell$ and $n$ produce the maximum overlap of the dressed state $\mid \psi_{i}^{\text {flux }}+$ res $\rangle=\left|\ell^{-}, n\right\rangle$ with the product state $|\ell, n\rangle$. As in Ref. [44], we are interested mainly in transitions among fluxonium states, where the quantum number $\ell$ changes. We, therefore, define the total transition rate due to the Purcell effect among fluxonium states as a sum over all possible initial and final states of the resonator, weighting initial states by their probability of being thermally occupied $P_{\text {res }}(n)=$ $\left[1-\exp \left(-\beta \hbar \omega_{r}\right)\right] \exp \left(-n \beta \hbar \omega_{r}\right)$. This sum yields

$$
\begin{aligned}
\Gamma_{\ell \rightarrow \ell^{\prime}}^{\text {Purcell } \uparrow}= & \sum_{n, n^{\prime}} P_{\text {res }}(n) \kappa n_{\mathrm{th}}\left(\omega_{\ell^{\prime}, n^{\prime}, \ell, n}\right) \\
& \times\left|\left\langle\overline{\ell^{\prime}, n^{\prime}}\left|a^{\dagger}\right| \overline{\ell, n}\right\rangle\right|^{2},
\end{aligned}
$$

for upward transitions, where $\omega_{\ell^{\prime}, n^{\prime}, \ell, n}=\left(E_{\ell^{\prime}, n^{\prime}}-E_{\ell, n}\right) / \hbar$, and

$$
\begin{aligned}
\Gamma_{\ell \rightarrow \ell^{\prime}}^{\text {Purcell } \downarrow}= & \sum_{n, n^{\prime}} P_{\text {res }}(n) \kappa\left[n_{\mathrm{th}}\left(-\omega_{\ell^{\prime}, n^{\prime}, \ell, n}\right)+1\right] \\
& \times\left|\left\langle\overline{\ell^{\prime}, n^{\prime}}|a| \overline{\ell, n}\right\rangle\right|^{2}
\end{aligned}
$$

for downward transitions.

The direct Purcell loss $(|e\rangle \rightarrow|g\rangle)$ gives a $T_{1}$ limit of approximately $100 \mathrm{~ms}$, effectively negligible in our experiments. However, heating to the excited levels of fluxonium due to the finite bath temperature results in enhanced Purcell loss. Some of these states (eighth, ninth, and tenth eigenstates) have transition frequencies from the logical manifold that are close to the resonator frequency, resulting in avoided crossings. While their exact location depends sensitively on the circuit parameters, these resonances are likely responsible for the decreased $T_{1}$ observed near 


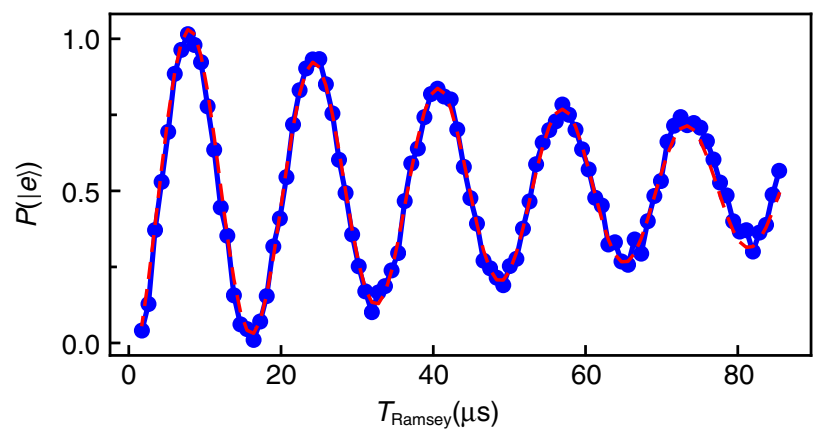

FIG. 9. Ramsey experiment at the flux-frustration point. The $T_{2}^{*}$ obtained by fitting the decay to an exponential is $71.3 \mu \mathrm{s}$.

$0.35 \Phi_{0}$. The total Purcell relaxation rate for a bath temperature of $60 \mathrm{mK}$ corresponds the dotted blue curve in Fig. 3(a) of the main text.

\section{APPENDIX H: MODELING FLUXONIUM DEPHASING}

On the flux slope, the decay envelope of a Ramsey experiment is best approximated by a Gaussian $\exp \left(-t^{2} / T_{\phi}^{2}\right)$, where $T_{\phi}=\Gamma_{\phi}^{-1}=\left[\sqrt{2} \eta\left(\partial_{\phi} \omega_{01}\right) \sqrt{\ln \omega_{i r} t}\right]^{-1}$ to first order. For the spin-echo experiments, low-frequency noise has a reduced weight in the noise spectrum, with $T_{\phi}=\left[\sqrt{W} \eta\left(\partial_{\phi} \omega_{01}\right)\right]^{-1}$. We can calculate $W$ for three echo $\pi$ pulses based on Ref. [32]. At the flux-frustration point, the qubit is first-order insensitive to $1 / f$ flux noise, and the spin-echo data can be explained with an exponential decay from white noise $\left(T_{2 e}=T_{C}=\Gamma_{C}^{-1}\right)$. In the regime of our spin-echo flux sweep, both noise sources contribute significantly. The data are, therefore, fit to a product of a Gaussian and an exponential [44], with the $T_{2 e}$ defined as $\exp \left(-T_{2 e} / T_{C}-T_{2 e}^{2} / T_{\phi}^{2}\right)=1 / e$, i.e.,

$$
T_{2 e}=\frac{\sqrt{1 / T_{C}^{2}+4 / T_{\phi}^{2}}-1 / T_{C}}{2 / T_{\phi}^{2}} .
$$

[1] M. H. Devoret and R. J. Schoelkopf, Superconducting Circuits for Quantum Information: An Outlook, Science 339, 1169 (2013).

[2] P. Krantz, M. Kjaergaard, F. Yan, T. P. Orlando, S. Gustavsson, and W. D. Oliver, A Quantum Engineer's Guide to Superconducting Qubits, Appl. Phys. Rev. 6, 021318 (2019).

[3] J. Preskill, Quantum Computing in the NISQ Era and Beyond, Quantum 2, 79 (2018).

[4] F. Arute, K. Arya, R. Babbush, D. Bacon, J. C. Bardin, R. Barends, R. Biswas, S. Boixo, F. G. Brandao, D. A. Buell et al., Quantum Supremacy Using a Programmable Superconducting Processor, Nature (London) 574, 505 (2019).
[5] H. Paik, D. I. Schuster, L. S. Bishop, G. Kirchmair, G. Catelani, A. P. Sears, B. R. Johnson, M. J. Reagor, L. Frunzio, L. I. Glazman, S. M. Girvin, M. H. Devoret, and R. J. Schoelkopf, Observation of High Coherence in Josephson Junction Qubits Measured in a Three-Dimensional Circuit QED Architecture, Phys. Rev. Lett. 107, 240501 (2011).

[6] J. M. Gambetta, C. E. Murray, Y.-K.-K. Fung, D. T. McClure, O. Dial, W. Shanks, J. W. Sleight, and M. Steffen, Investigating Surface Loss Effects in Superconducting Transmon Qubits, IEEE Trans. Appl. Supercond. 27, 1 (2016).

[7] A. Dunsworth, A. Megrant, C. Quintana, Z. Chen, R. Barends, B. Burkett, B. Foxen, Y. Chen, B. Chiaro, A. Fowler et al., Characterization and Reduction of Capacitive Loss Induced by Sub-micron Josephson Junction Fabrication in Superconducting Qubits, Appl. Phys. Lett. 111, 022601 (2017).

[8] Y. Nakamura, Y. A. Pashkin, and J. S. Tsai, Coherent Control of Macroscopic Quantum States in a SingleCooper-Pair Box, Nature (London) 398, 786 (1999).

[9] W. D. Oliver, Mach-Zehnder Interferometry in a Strongly Driven Superconducting Qubit, Science 310, 1653 (2005).

[10] D. M. Berns, W. D. Oliver, S. O. Valenzuela, A. V. Shytov, K. K. Berggren, L. S. Levitov, and T. P. Orlando, Coherent Quasiclassical Dynamics of a Persistent Current Qubit, Phys. Rev. Lett. 97, 150502 (2006).

[11] C. Hicke, L. F. Santos, and M. I. Dykman, Fault-Tolerant Landau-Zener Quantum Gates, Phys. Rev. A 73, 012342 (2006).

[12] D. L. Campbell, Y.-P. Shim, B. Kannan, R. Winik, A. Melville, B. M. Niedzielski, J. L. Yoder, C. Tahan, S. Gustavsson, and W.D. Oliver, Universal Nonadiabatic Control of Small-Gap Superconducting Qubits, arXiv: 2003.13154 [Phys. Rev. X (to be published)].

[13] N. Earnest, S. Chakram, Y. Lu, N. Irons, R. K. Naik, N. Leung, L. Ocola, D. A. Czaplewski, B. Baker, J. Lawrence, J. Koch, and D. I. Schuster, Realization of a $\Lambda$ System with Metastable States of a Capacitively Shunted Fluxonium, Phys. Rev. Lett. 120, 150504 (2018).

[14] Y.-H. Lin, L. B. Nguyen, N. Grabon, J. S. Miguel, N. Pankratova, and V.E. Manucharyan, Demonstration of Protection of a Superconducting Qubit from Energy Decay, Phys. Rev. Lett. 120, 150503 (2018).

[15] J. Koch, T. M. Yu, J. Gambetta, A. A. Houck, D. I. Schuster, J. Majer, A. Blais, M. H. Devoret, S. M. Girvin, and R. J. Schoelkopf, Charge-Insensitive Qubit Design Derived from the Cooper Pair Box, Phys. Rev. A 76, 042319 (2007).

[16] A. Nersisyan, S. Poletto, N. Alidoust, R. Manenti, R. Renzas, C.-V. Bui, K. Vu, T. Whyland, Y. Mohan, E. A. Sete et al., Manufacturing Low Dissipation Superconducting Quantum Processors, arXiv:1901.08042.

[17] K. X. Wei, I. Lauer, S. Srinivasan, N. Sundaresan, D. T. McClure, D. Toyli, D. C. McKay, J. M. Gambetta, and S. Sheldon, Verifying Multipartite Entangled GHZ States via Multiple Quantum Coherences, Phys. Rev. A 101, 032343 (2020).

[18] J. Mooij, T. Orlando, L. Levitov, L. Tian, C. H. Van der Wal, and S. Lloyd, Josephson Persistent-Current Qubit, Science 285, 1036 (1999). 
[19] I. Chiorescu, Y. Nakamura, C. M. Harmans, and J. Mooij, Coherent Quantum Dynamics of a Superconducting Flux Qubit, Science 299, 1869 (2003).

[20] I. Chiorescu, P. Bertet, K. Semba, Y. Nakamura, C. Harmans, and J. Mooij, Coherent Dynamics of a Flux Qubit Coupled to a Harmonic Oscillator, Nature (London) 431, 159 (2004).

[21] F. Yan, S. Gustavsson, A. Kamal, J. Birenbaum, A. P. Sears, D. Hover, T. J. Gudmundsen, D. Rosenberg, G. Samach, S. Weber et al., The Flux Qubit Revisited to Enhance Coherence and Reproducibility, Nat. Commun. 7, 12964 (2016).

[22] V. E. Manucharyan, J. Koch, L. I. Glazman, and M. H. Devoret, Fluxonium: Single Cooper-Pair Circuit Free of Charge Offsets, Science 326, 113 (2009).

[23] V. E. Manucharyan, N. A. Masluk, A. Kamal, J. Koch, L. I. Glazman, and M.H. Devoret, Evidence for Coherent Quantum Phase Slips across a Josephson Junction Array, Phys. Rev. B 85, 024521 (2012).

[24] I. M. Pop, K. Geerlings, G. Catelani, R. J. Schoelkopf, L. I. Glazman, and M.H. Devoret, Coherent Suppression of Electromagnetic Dissipation due to Superconducting Quasiparticles, Nature (London) 508, 369 (2014).

[25] U. Vool, I. M. Pop, K. Sliwa, B. Abdo, C. Wang, T. Brecht, Y. Y. Gao, S. Shankar, M. Hatridge, G. Catelani et al., NonPoissonian Quantum Jumps of a Fluxonium Qubit due to Quasiparticle Excitations, Phys. Rev. Lett. 113, 247001 (2014).

[26] L. B. Nguyen, Y.-H. Lin, A. Somoroff, R. Mencia, N. Grabon, and V.E. Manucharyan, High-Coherence Fluxonium Qubit, Phys. Rev. X 9, 041041 (2019).

[27] U. Vool, A. Kou, W. C. Smith, N. E. Frattini, K. Serniak, P. Reinhold, I. M. Pop, S. Shankar, L. Frunzio, S. M. Girvin, and M. H. Devoret, Driving Forbidden Transitions in the Fluxonium Artificial Atom, Phys. Rev. Applied 9, 054046 (2018).

[28] V. E. Manucharyan, J. Koch, M. Brink, L. I. Glazman, and M. H. Devoret, Coherent Oscillations between Classically Separable Quantum States of a Superconducting Loop, arXiv:0910.3039.

[29] P. Magnard, P. Kurpiers, B. Royer, T. Walter, J.-C. Besse, S. Gasparinetti, M. Pechal, J. Heinsoo, S. Storz, A. Blais, and A. Wallraff, Fast and Unconditional All-Microwave Reset of a Superconducting Qubit, Phys. Rev. Lett. 121, 060502 (2018).

[30] A. Wallraff, D. I. Schuster, A. Blais, L. Frunzio, R.-S. Huang, J. Majer, S. Kumar, S. M. Girvin, and R. J. Schoelkopf, Strong Coupling of a Single Photon to a Superconducting Qubit Using Circuit Quantum Electrodynamics, Nature (London) 431, 162 (2004).

[31] G. Zhu, D. G. Ferguson, V. E. Manucharyan, and J. Koch, Circuit QED with Fluxonium Qubits: Theory of the Dispersive Regime, Phys. Rev. B 87, 024510 (2013).

[32] G. Ithier, E. Collin, P. Joyez, P. J. Meeson, D. Vion, D. Esteve, F. Chiarello, A. Shnirman, Y. Makhlin, J. Schriefl, and G. Schön, Decoherence in a Superconducting Quantum Bit Circuit, Phys. Rev. B 72, 134519 (2005).
[33] M. A. Rol, F. Battistel, F. K. Malinowski, C. C. Bultink, B. M. Tarasinski, R. Vollmer, N. Haider, N. Muthusubramanian, A. Bruno, B. M. Terhal, and L. DiCarlo, Fast, High-Fidelity Conditional-Phase Gate Exploiting Leakage Interference in Weakly Anharmonic Superconducting Qubits, Phys. Rev. Lett. 123, 120502 (2019).

[34] E. Magesan, J. M. Gambetta, B. R. Johnson, C. A. Ryan, J. M. Chow, S. T. Merkel, M. P. da Silva, G. A. Keefe, M. B. Rothwell, T. A. Ohki, M. B. Ketchen, and M. Steffen, Efficient Measurement of Quantum Gate Error by Interleaved Randomized Benchmarking, Phys. Rev. Lett. 109, 080505 (2012).

[35] E. Knill, D. Leibfried, R. Reichle, J. Britton, R. B. Blakestad, J. D. Jost, C. Langer, R. Ozeri, S. Seidelin, and D. J. Wineland, Randomized Benchmarking of Quantum Gates, Phys. Rev. A 77, 012307 (2008).

[36] J. M. Chow, J. M. Gambetta, L. Tornberg, J. Koch, L. S. Bishop, A. A. Houck, B. R. Johnson, L. Frunzio, S. M. Girvin, and R. J. Schoelkopf, Randomized Benchmarking and Process Tomography for Gate Errors in a Solid-State Qubit, Phys. Rev. Lett. 102, 090502 (2009).

[37] A. P. M. Place, L. V. H. Rodgers, P. Mundada, B. M. Smitham, M. Fitzpatrick, Z. Leng, A. Premkumar, J. Bryon, S. Sussman, G. Cheng, T. Madhavan, H. K. Babla, B. Jaeck, A. Gyenis, N. Yao, R. J. Cava, N. P. de Leon, and A. A. Houck, New Material Platform for Superconducting Transmon Qubits with Coherence Times Exceeding 0.3 Milliseconds, arXiv:2003.00024.

[38] K. N. Nesterov, I. V. Pechenezhskiy, C. Wang, V.E. Manucharyan, and M. G. Vavilov, Microwave-Activated Controlled-z Gate for Fixed-Frequency Fluxonium Qubits, Phys. Rev. A 98, 030301(R) (2018).

[39] R. Vijay, D. H. Slichter, and I. Siddiqi, Observation of Quantum Jumps in a Superconducting Artificial Atom, Phys. Rev. Lett. 106, 110502 (2011).

[40] M. Hatridge, S. Shankar, M. Mirrahimi, F. Schackert, K. Geerlings, T. Brecht, K. M. Sliwa, B. Abdo, L. Frunzio, S. M. Girvin, R. J. Schoelkopf, and M. H. Devoret, Quantum Back-action of an Individual Variable-Strength Measurement, Science 339, 178 (2013).

[41] J. Johansson, P. Nation, and F. Nori, Qutip 2: A РYтноN Framework for the Dynamics of Open Quantum Systems, Comput. Phys. Commun. 184, 1234 (2013).

[42] R. Schoelkopf, A. Clerk, S. Girvin, K. Lehnert, and M. Devoret, Qubits as Spectrometers of Quantum Noise, in Quantum Noise in Mesoscopic Physics (Springer, New York, 2003), pp. 175-203.

[43] A. A. Clerk, M. H. Devoret, S. M. Girvin, F. Marquardt, and R. J. Schoelkopf, Introduction to Quantum Noise, Measurement, and Amplification, Rev. Mod. Phys. 82, 1155 (2010).

[44] P. Groszkowski, A. D. Paolo, A. L. Grimsmo, A. Blais, D. I. Schuster, A. A. Houck, and J. Koch, Coherence Properties of the $0-\pi$ Qubit, New J. Phys. 20, 043053 (2018). 